Christophe Lebigre ${ }^{1}$, Catherine Timmermans ${ }^{2,3,4}$, Carl D. Soulsbury ${ }^{3}$

\title{
NO BEHAVIOURAL RESPONSE TO KIN COMPETITION IN A LEKKING SPECIES
}

${ }^{1}$ Earth and Life Institute, Catholic University of Louvain, Belgium (1)

${ }^{2}$ Institute of Statistics, Biostatistics and Actuarial Sciences, Catholic University of Louvain, Belgium

${ }^{3}$ Joseph Banks Laboratories, School of Life Sciences, University of Lincoln, Lincoln, Lincolnshire, LN6 7TS, UK.

${ }^{4}$ Department of Mathematics, University of Liège, Belgium

Corresponding author:

Christophe Lebigre

Email: christophe.lebigre@uclouvain.be

Phone: +32 10479228 (office)

Acknowledgments: This paper is dedicated to Prof. Rauno Veli Alatalo who passed away on November $9^{\text {th }}$ 2012. We thank Jefferson Graves, Matti Kervinen and two anonymous reviewers for their insightful comments on previous versions of this manuscript. We are grateful to Elina Virtanen, Juho Niva, Anssi Lipponen, Sami Kyröläinen, and Henna Ojaniemi for help in the lab. This project was founded by the Academy of Finland (Grant nos. 7211271 and 7119165) and a fellowship of the Belgian Fond National pour la Recherche Scientifique (FNRS). Part of the statistical analysis was conducted within the SMCS (Support en Méthodologie et Calcul Statistique - Université Catholique de Louvain). 


\section{Abstract}

The processes of kin selection and competition may occur simultaneously if limited individual dispersal i.e. population viscosity, is the only cause of the interactions between kin. Therefore, the net indirect benefits of a specific behaviour may largely depend on the existence of mechanisms dampening the fitness costs of competing with kin. In lekking species, males may increase the mating success of their close relatives (and hence gain indirect fitness benefits) because female prefer large leks. At the same time, kin selection may also lead to the evolution of mechanisms that dampen the costs of kin competition. As this mechanism has largely been ignored to date, we used detailed behavioural and genetic data collected in the black grouse Lyrurus tetrix to test whether males mitigate the costs of kin competition through the modulation of their fighting behaviours according to kinship and the avoidance of close relatives when establishing a lek territory. We found that neighbouring males' fighting behaviour was unrelated to kinship and males did not avoid settling down with close relatives on leks. As males' current and future mating success are strongly related to their behaviour on the lek (including fighting behaviour and territory position), the costs of kin competition may be negligible relative to the direct benefits of successful male-male contests. As we previously showed that the indirect fitness benefits of group membership were very limited in this black grouse population, these behavioural data support the idea that direct fitness benefits gained by successful male-male encounters likely outbalance any indirect fitness benefits.

Keywords: dominance, indirect fitness benefits, kin selection, kin competition, territoriality, sexual selection, sociality 


\section{Significance statement}

54 Kin selection might be involved in the formation of groups because the fitness benefits of increasing group size can be accrued when groups hold close relatives. However, the fitness costs of competing with kin could counter-balance these indirect fitness benefits unless mechanisms enabling individuals to limit kin competition. Using data collected in the black grouse (Lyrurus tetrix) we show that males do not modulate their fight frequency and

59 intensity according to their kinship and do not avoid establishing territories with closely related neighbours. As we previously showed that the indirect fitness benefits of group display were very small and as this study shows that males do not show any sign of kin competition avoidance, the indirect effects associated with male group display are likely to be very small in this system. 


\section{Introduction}

Kinship among group members influences multiple aspects of animal societies ranging from individual interactions to group formation. Indeed, individuals can behave in ways favouring kin (Brown and Brown 1996; Silk 2002), such as increasing their helping rate according to kinship (Reeve et al. 1990; Komdeur 1994; Russell and Hatchwell 2001), forming mating or foraging alliances (Russell and Hatchwell 2001; Krützen et al. 2003; Krakauer 2005;

Piertney et al. 2008; Edenbrow and Croft 2012), or showing reduced aggressiveness towards kin (Silk 2002; Smith et al. 2010; though see West et al. 2001). Individuals may also benefit kin more indirectly, by avoiding interacting with kin by dispersing (Moore et al. 2006; Bitume et al. 2013), or through the avoidance of groups holding close relatives (Höner et al. 2007). As many of these behaviours can co-occur, the adaptive value of a specific behaviour can only fully be understood when the indirect costs and benefits (defining kin selection and competition) resulting from these behaviour can be estimated (Hamilton 1964; Griffin and West 2002; Grafen 2006).

In lekking species, males gather on specific areas to display on territories visited by females for the sole purpose of mating (Höglund and Alatalo 1995). Lekking males are expected to choose their lek site according to their display abilities (e.g. Alatalo et al. 1992) and a large number of morphological and behavioural traits has been associated with male mating success (Höglund and Alatalo 1995; Fiske et al. 1998). By joining large leks, males might gain both direct fitness benefits (the number of observed copulations increases with lek size, Isvaran and Ponkshe 2013; Lebigre et al. 2014) and indirect fitness benefits when leks comprise close relatives (males may increase the mating opportunities of their close relatives; Kokko and Lindström 1996; Höglund 2003; Hatchwell 2010). Several studies have tested whether leks comprised closely related individuals either by quantifying the mean relatedness across lekking males (e.g. Bouzat and Johnson 2004) or by measuring the spatial aggregation of close relatives within (e.g. Shorey et al. 2000; Segelbacher et al. 2007) and among leks (Höglund et al. 1999). To our knowledge, only one study quantified the 
indirect fitness benefits resulting from male aggregations and showed that these were very limited and substantially less than male direct fitness benefits (Lebigre et al. 2014). However, theory predicts that if population viscosity (i.e. limited dispersal) is the sole driver of the interaction between kin, the indirect fitness costs associated with individuals' action may reduce or even cancel out all indirect fitness benefits (e.g. West-Eberhard 1975; Taylor 1992; Wilson et al. 1992; Van Dyken 2010) and kin selection may only matter in systems where it has also led to the evolution of mechanisms reducing kin competition (Mitteldorf and Wilson 2000; Alizon and Taylor 2008; Lion and Gandon 2009).

Indirect fitness costs are required in order to characterise kin competition. Yet, stable dominance hierarchies may reduce the costs of aggressive encounters (Berglund et al. 1996; Hsu et al. 2006) even in lekking species (Magaña et al. 2011). However, such a mitigation may largely be counter-balanced in lekking species by female preference for male fighting behaviour itself (Höglund et al. 1997; Hämäläinen et al. 2012). Males fighting behaviour could therefore be an honest indicator of male quality either directly (Briffa and Sneddon 2007) or indirectly through males' ability to maintain intact ornaments during the lekking season (Kirkpatrick and Ryan 1991; Höglund et al. 1994). Lekking is also energetically very costly (Vehrencamp et al. 1989; Höglund et al. 1992) and these energetic costs may lead to fitness costs depending on individuals' age and phenotypic quality (Gosling et al. 1987; McElligott et al. 2001; 2003; Kervinen et al. 2015; 2016). Therefore, the intense and direct competition observed in lekking species may lead to indirect fitness costs when males are displaying with kin either through a reduced attractiveness or a decreased survival likelihood. Nevertheless, the degree to which kin selection can lead to the evolution of a reduction of kin competition in lekking species has largely been overlooked. For instance, studies failing to report strong kin structure (e.g. Gibson et al. 2005; Loiselle et al. 2007; Lebigre et al. 2008) interpreted their results as indicative of an absence of kin selection, while individuals may simply avoid competing with close relatives. 
We used data collected in a classical lekking species, the black grouse (Lyrurus tetrix), to determine whether kin selection can have led to the evolution of two mechanisms dampening the costs of kin competition: the modulation of aggressive interactions between close relatives and the avoidance of territories with closely related neighbours. To this end, we combined behavioural data (territory positions, fighting rate and intensity) with measures of male kinship and conducted a twofold analysis. In this species, the competition with kin may lead to fitness costs as it has previously been shown that lekking is energetically costly (Lebigre et al. 2013), that male fighting behaviour is under direct sexual selection (Höglund et al. 1997; Hämäläinen et al. 2012; Kervinen et al. 2016) and that male's ability to maintain high quality ornaments is related to their mating success (Alatalo et al. 1991; Höglund et al. 1994). First we measured the relatedness between neighbouring territorial males and tested whether males fought less frequently and less intensively with closely related neighbours. Such type of analysis based is not straightforward as variables such as the fight frequency and intensity within a group are likely to have a spatial structure. Indeed, the fight frequency between two males is influenced by and influences their fight frequency with their other neighbours (i.e. if "A" fights with "B", "B" cannot fight with its neighbour "C") and similarly the intensity of male fights may be lower with specific neighbours if the dominance hierarchy is well established. Such dependence structures may result in a spatial correlation which needs to be explicitly accounted for in a mixed model. Yet, contrary to the usual spatial correlation models used in e.g. geostatistics, the proximity between individuals should not be measured in terms of geographical distance per se but in terms of neighbourhood. Therefore, we used the identity of neighbouring males to define a network in which each bird is a node and the proximity between birds as measured as the number of edges separating them (a measure named "n-hop distance").

Second, we determined whether males avoided settling on territories with closely related neighbours using a randomisation approach. Like in many other territorial species, male territory positions are dynamic in the black grouse. Newcomers generally display on the 
lek periphery and slowly move towards the lek centre as a consequence of shifts of territory positions and the arrival of other more peripheral males (Kokko et al. 1997, 1999). We therefore conducted a spatially constrained randomisation test in which a set of potentially available territories was defined (i.e. the territories of all newly established males and other very subordinate males). This enabled us to test the hypothesis that new territorial males (newcomers) established their territory with less closely related neighbours than expected by chance.

\section{Material and Methods}

\section{Study population}

The data used in this study were collected in a black grouse population inhabiting Central Finland (2003-2005). Upon capture, all males were ringed with an aluminium ring and a unique combination of colour rings for future identification. Birds were trapped in several sites but here we will focus on three sites (Kummunsuo, Valkeissuo, Teerijärvensuo) where 95\% of the lekking males were ringed ( $N=78$ unique individuals for 115 observations; some males were observed in several years and others had no neighbours, Suppl. Table 1). The distance between these study sites (range 23.02-36.52 km) exceeds the current recorded maximum natal dispersal distance in this species $(11 \mathrm{~km})$ while the vast majority of the males remain in their natal area (Caizergues and Ellison 2002; Warren and Baines 2002).

Therefore, the study sites can be considered as separate entities with infrequent movements between them. A small blood sample was taken from the birds' brachial vein from which DNA was extracted and all individuals were genotyped at 11 microsatellite loci (detailed description in Lebigre et al. 2007). We measured individuals' pairwise relatedness using Queller and Goodnight's estimator (RQG, Queller and Goodnight 1989; details in Lebigre et al. 2008) and more conservatively identified close relatives as having a value of $R_{Q G}$ over 0.2 . 
This cut-off value was chosen because it enabled us to limit the risks of wrongly identifying unrelated dyads are close relatives (details in Lebigre et al. 2010, 2014).

\section{Lek observations}

Male-male interactions were recorded during ca. 10 days at the end of April-early May when nearly all copulations take place (Lebigre et al. 2007). During the lekking season, males gather on various open areas such as peat bogs, frozen lakes and forest clear-cuts to defend a small territory where they display (Hovi et al. 1994; Höglund and Alatalo 1995). Male lek activity was recorded on behavioural maps every day during the most active lekking days (ca. 10 days). Maps were drawn every 5 minutes (depending on lek size) from ca. 03:00 to 09:00 with males' exact position and a description of its behaviour categorised as inactive, rookooing (main vocalisation), hissing (occasional loud scream) and fighting. When fights occurred, the identities of the two males was recorded as well as the fight intensity (three levels; Hämäläinen et al. 2012). Male attendance to the lek was calculated as the proportion of maps drawn on which a specific male is recorded relative to total the number of maps drawn for the most attending male (Rintamäki et al. 2001). Males were considered territorial when having an attendance to the lek $>0.3$ meaning that their total number of recorded activity was at least $30 \%$ of that of the most attending male of the lek (see Kervinen et al. 2012). The position of the territory of each male was calculated as the median of all $x$ and $y$ coordinates of the recorded observations and all observations were plotted to delineate territory boundaries and identify neighbours (Suppl. Fig. 1). This also allowed us to locate ditches in peat harvested sites which effectively prevent the interaction between neighbouring males (males were not considered as neighbours if a ditch delineated the boundary of their territories). The lek centre was defined as the median of all $x$ and $y$ coordinates across all males. We then calculated the Euclidian distance separating each male's territory to the lek centre to estimate male's territory centrality. For each unique pair of neighbours in each year ( $N=195$ from the 78 unique individuals), we calculated the proportion of observations in which neighbours were fighting (i.e. the fight frequency) and the 
median intensity of the fights (i.e. fight intensity). Fights occasionally involving nonneighbouring males were excluded from the analyses as they occurred when males left their territories to feed or approach females. This study combines two dataset which were collected independently. In the field, it was not possible to record data blind because we used marked birds with colour rings. However, only part of the birds' unique identification number was used during the genotyping which was carried out with no knowledge of the lekking behaviour of the males and the location of their lek territories.

\section{Statistical analyses}

In all analyses we used two measures of relatedness: the direct measures of $R_{Q G}(a$ continuous and normally distributed measure of genetic distance) and a binary variable describing whether individuals were close relatives $\left(R_{Q G}>0.2\right)$ or not (this variable is denoted $\left.R_{Q G \_b i n a r y}\right)$. We used $R_{Q G \_b i n a r y}$ because if individuals really avoid competing with kin, these effects will be easier to detect among close relatives. We tested whether the relatedness between neighbouring males influenced their fight frequency and fight intensity. Those two cases where considered successively, with slightly different statistical tools.

To test the hypothesis that male fight frequency is influenced by their relatedness, we fitted a linear mixed model explaining the fight frequency between two neighbouring males as

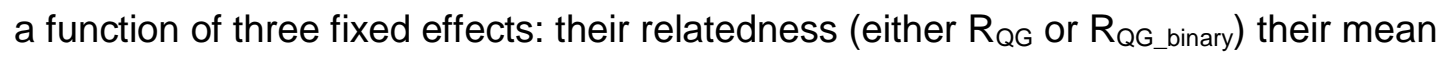
centrality and centrality difference. The two last fixed effects were used to control for the directionality of male-male interactions as we expected males closer to the lek centre (low mean centrality) and males having similar distances from the lek centre (low centrality difference) to be more active. The model also accounts for the fact that the baseline fight frequency is a priori lek-dependent and they are related to lek size by including each lek*year combination as a random effect. This implies that we assume that the effects of the pairs' relatedness, mean centrality, and centrality difference on the variance in fight frequency are 
not lek-specific. The mixed models are estimated using a simple retricted maximum likelihood estimator implemented using the R-package nlme (Pinheiro et al. 2013).

Two adaptations of the models are required to ensure their statistical validity. First, the fight frequency was log-transformed to produce normally distributed and homogeneous residuals. Second, we needed to account for the spatial structure resulting from the nonindependence of the interactions between neighbours and its potential cascading effects across the entire leks. Therefore, we tested whether the residuals of the models were spatially correlated. As the geographic distance is not the important parameter here, but rather the neighbourhood, we used the n-hop distance on a graph to describe the spatial structure instead of the Euclidean distance. The graph was built with birds as nodes, and undirected edges between each pair if birds were neighbours and the linear model is thus defined for estimating the fight frequency at each edge. The n-hop distance between two edges was computed as the number of nodes between them. Hence, a n-hop distance equal to one between two pairs of neighbours means that one individual is involved in the two pairs. The n-hop distances were calculated using the r-package using the r-package spa (Culp 2015). As expected, we found that there was a negative correlation in the model residuals for neighbouring males $(r=-0.100 ; P=0.045$; Suppl. Table 2$)$ meaning that a male fighting often with one neighbour fought less often with his other neighbours. To account for this spatial structure, we re-implemented the mixed effects models including a first order correlation of the residuals on the graph. P-values of the fixed effects and their confidence intervals were computed using a student statistics (more details in Suppl. Appendix 1).

We replicated these steps to test the hypothesis that fight intensity was influenced by the relatedness of the males. In all the analyses of male fight intensity, the dependent variable (median fight intensity) was transformed to a binary variable separating pairs fighting intensely (median intensity $\geq 2$ ) from the others. We therefore implemented generalised linear mixed effects models with a logit link function and a restricted maximum likelihood estimator. These models included three fixed effects (the pair relatedness measured either 


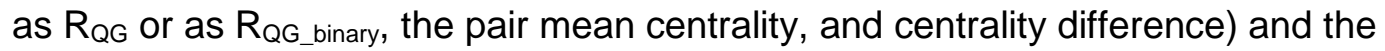
combination of lek* year as a random factor. These models were estimated using the Rpackage Ime4 (Bates et al. 2015). As there was no significant spatial structure for fight intensity we directly used the outcome of the generalised linear mixed effects models.

To test the hypothesis that lekking males could reduce kin competition by settling down on territories where they have no relatives, we focussed on newcomers. Newcomers are males which were captured as yearlings for whom we can therefore ensure that they defended a lek territory for the first time (newcomers are usually one or two years old; $\mathrm{N}=$ 34). We first identified the location and territories boundaries of all newcomers, measured their $\mathrm{R}_{\mathrm{QG}}$ to their neighbours and to the other lekking males. We then used a mixed model to determine whether males were less related to their neighbours than to the other lekking males using the mean $R_{Q G}$ as the dependent variable, a binary variable describing whether the mean was calculated across neighbouring or non-neighbouring males. Male identity was set as a random factor nested within each year*lek combination. In addition to these paired comparisons, we carried out a randomisation test to determine whether males settled down on a territory with less closely related neighbours than expected by chance. We first identified potential territories where males could have settled down as those occupied by other newcomers and peripheral territories. We then calculated the mean $\mathrm{R}_{Q \mathrm{G}}$ and number of neighbouring close relatives newcomer would have had if they had occupied one of these potential territories. We used the software PopTools $3.2(\operatorname{Hood} 2011)$ to shuffle each newcomer to each of the potential territories. We ran 500 iterations to generate random distributions for the mean relatedness between neighbours and the number of closely related neighbours. The observed mean relatedness to the neighbours and number of close relatives were then compared to these random distributions and hence determine whether males settled down with less closely related neighbours than expected by chance. 


\section{Results}

275

276

277

Across all leks and years $83 \%$ of the neighbouring males were unrelated (mean $R_{Q G}=0.02 \pm$ $0.18(\mathrm{SD}), \mathrm{N}_{\mathrm{Tot}}=195$, Fig. 1a) and most males had no closely related neighbour (Fig. 1b). The fight frequency and intensity between neighbouring males were not influenced by their relatedness whether it was measured $R_{Q G}$ between neighbours nor $R_{Q G \_b i n a r y}(T a b l e ~ 1)$. The only factor which significantly influenced fight frequency and intensity was the difference in territory centrality as fights were more frequent and more intense at small differences in territory centrality (Table 1, Fig. 2).

Across newcomers, there was no significant difference between the mean $R_{Q G}$ of neighbours and non-neighbours $(\beta=-0.023 \pm 0.018(S E) ; t=-1.254 ; P=0.221 ;$ Fig. 3$)$. The spatially constrained randomisation test showed that newcomers were not less related to their neighbours than expected by chance (mean observed $R_{Q G}=0.02 \pm 0.11(S D), N_{\text {Newcomers }}$ $=34$, mean expected $R_{Q G}=0.00 \pm 0.02$ (SD), $P=0.24$, Suppl. Fig. 2). Similarly, the observed mean proportion of close relative neighbours (i.e. $R_{Q G}>0.2$ ) did not differ from the random expectation (observed mean proportion neighbouring close relatives $=0.166, \mathrm{~N}=34$; expected mean proportion neighbouring close relatives $=0.137 \pm 0.033(\mathrm{SD}), \mathrm{P}=0.19$ ).

\section{Discussion}

As kin selection and competition may occur simultaneously, the overall fitness consequences of the interactions between close relatives may depend on an adaptive response dampening the fitness costs of kin competition. We addressed this knowledge gap by testing whether kin selection could have led to the evolution of the two main behavioural mechanisms through which males may mitigate kin competition: a decrease in fight frequency/intensity and the avoidance of closely related neighbours. We found no evidence of these mechanisms in the studied black grouse leks. 
As males in lekking species defend clustered territories, one obvious way to potentially reduce kin competition is to reduce the aggressive interactions with close relatives. Such a process has been reported in many taxa (e.g. Koprowski 1996; Pravosudova 2001; Wahaj et al. 2004; Smith et al. 2010), including a lekking species (satin bowerbirds, Ptilonorhynchus violaceus; Reynolds et al. 2008) in which a decrease in aggression was found between closely related individuals which facilitated the formation of kin clusters . In our study, the frequency and intensity of fights between neighbours in black grouse were unrelated to their relatedness. Male black grouse spend a large proportion of their time fighting (Hämäläinen et al. 2012), whereas in satin bowerbirds, individuals rarely directly interact and the cooperative behaviours of wild turkeys males is a rare and remarkable feature for a lekking species (Höglund and Alatalo 1995). Black grouse males form tightly clustered leks on which females prefer dominant males defending central territories and expressing a wide variety of behavioural and morphological traits (see Kervinen et al. 2015, 2016). As male mating success is also strongly related to males' past lekking performance (Kokko et al. 1997, 1999) and current fighting performance and frequency (Lebigre et al. 2012; Hämäläinen et al. 2012), the benefits of the modulation of their behaviour with relatedness may be negligible compared with the direct benefits of successful male-male contests. Furthermore, there were very few closely related neighbours meaning that males may actually not even need to modulate their behaviour to reduce the fitness costs of kin competition. In lekking species where no kin structures have been found (and hence closely related neighbours are probably rare), relatedness-related changes in display behaviour are also unlikely but this remains to be tested (e.g. Gibson et al. 2005; Loiselle et al. 2007). In studies where kin structures have been found, it is critical to undertake similar analyses to better understand the importance of kin selection as male philopatry is the main factor explaining the formation of these kin groups and males indirect fitness benefits may actually be substantially larger if they also reduce their level of aggression (e.g. Shorey et al. 2000; Segelbacher et al. 2007). Even if in our case the influence of the spatial structure of the males did not strongly influence males' fight frequency 
and intensity we stress that applying a similar approach is the most appropriate way to account for a spatial structure for which the neighbourhood distances are more relevant than geographical distances per se.

The lack of closely related neighbours may result from the active avoidance of close relatives when males establish their lek territories. However, we found no evidence that the territory location of newcomer males to the leks was not random with respect to the relatedness of the neighbouring males. This might again be due to the low likelihood of settling down on a lek holding close relatives in this large continuous population (Lebigre et al. 2008,2014 ) but also be due to the other factors that influence the spatial arrangements of the territories. Indeed, newcomers may have limited control over the identity of their neighbours and location of their territory given their lack of prior lek experience in pairwise conflicts (Templeton et al. 2012) and their lower competitive abilities (Parker and Sutherland 1986; Alatalo et al. 1992). More specifically, information concerning the past territory positions (Kokko et al. 1999) and the physical strength and ornament expression that determine male dominance (Kervinen et al. 2012, 2015, 2016) may be far more important for the current and future dominance status (and hence fitness) of the newcomer males than their relatedness to the neighbours. Territorial birds may tolerate kin and facilitate their recruitment in the local populations through the older males' secession of part of their territories (e.g. Piertney et al. 1999; MacColl et al. 2000; Piertney et al. 2008). In black grouse, the most successful males defend central territories and therefore are unlikely to display close to their sons who might only be able to defend peripheral territories (Kokko et al. 1997). Yet, it might be interesting to examine the temporal changes in both territory position and neighbours identity to determine the stability of the neighbourhoods and dominance hierarchies which can strongly influence males' lek performance and their mating success.

When males establish their lek territory, they will have three critical choices to makes: which lek to join, when to join it, where to establish a territory. We have been able to show 
354 that kinship to the other lek member does not contribute substantially to these steps in black

355 grouse as leks are random subsets of the larger winter flocks (Lebigre et al. 2008), overall

356 males joining leks have limited indirect fitness benefits (Lebigre et al. 2014), and in this

357 study, we found that males did not avoid fighting with close relatives and the location of

358 newcomers' territories was not influenced by its relatedness to its neighbours. Therefore, it is

359 now important to determine whether such a lack of behavioural response to competitive

360 interactions with kin are consistent over time and space. We showed that the indirect fitness

361 benefits gained by the lekking males depend on population density (Lebigre et al. 2014). In a

362 similar fashion, behavioural adjustments may also be easier to detect when the indirect costs

363 of competing with kin are high and female visits to the leks are rare (e.g. low population

364 density years). Moreover, it is now critical to try to quantify both the indirect fitness costs and

365 benefits and integrate them over individuals' lifespan to fully understand the net indirect

366 effects associated with male group display to fully quantify males' inclusive fitness and

367 determine the relative contribution of direct and indirect fitness components. The behavioural

368 data used in this study therefore imply that the direct fitness benefits gained by successful

369 male-male aggressive encounters likely outbalance indirect fitness benefits. 
372 Compliance with Ethical Standards

373 - Funding: This study was funded by the Academy of Finland (grant numbers 7211271 and

374 7119165), the Finnish Centre of Excellence in Evolutionary Research (211271), and the

375 Fond National de la Recherche Scientifique (FNRS A4/5 - MCF/DM).

376

377 - Conflict of Interest: CL declares that he has no conflict of interest. CT declares that she has

378 no conflict of interest. CDS declares that he has no conflict of interest.

379

380 - Ethical approval: All applicable international, national, and/or institutional guidelines for the

381 care and use of animals were followed. This work was carried out with the permission of

382 Central Finland Environmental Centre and the Animal Care Committee of the University of 383 Jyväskylä (permissions KSU-2003-L-25/254 and KSU-2002-L-4/254). This article does not 384 contain any studies with human participants performed by any of the authors. 


\section{References}

387

Alatalo RV, Höglund J, Lundberg A, Sutherland WJ (1992) Evolution of black grouse leks: female preferences benefit males in larger leks. Behav Ecol 3:53-59

Alizon S, Taylor P (2008) Empty sites can promote altruistic behavior. Evolution 62:13351344

Bates D, Maechler M, Bolker B, Walker S (2015) Fitting linear mixed-effects models using Ime4. J Stat Softw 67:1-48

Berglund A, Bisazza A, Pilastro A (1996) Armaments and ornaments: An evolutionary explanation of traits of dual utility. Biol J Linn Soc 58:385-399

Bitume EV, Bonte D, Ronce O, Bach F, Flaven E, Olivieri I, Nieberding CM (2013) Density and genetic relatedness increase dispersal distance in a subsocial organism. Ecol Lett 16:430-437

Bouzat JL, Johnson K (2004) Genetic structure among closely spaced leks in a peripheral population of lesser prairie-chickens. Mol Ecol 13:499-505

Briffa M, Sneddon LU (2007) Physiological constraints on contest behaviour. Funct Ecol $21: 627-637$

Brown GE, Brown JA (1996) Kin discrimination in salmonids. Rev Fish Biol Fisher 6:201-219

Caizergues A, Ellison LN (2002) Natal dispersal and its consequences in Black Grouse Tetrao tetrix. Ibis 144:478-487

Culp M (2011) spa: a semi-supervised R package for semi-parametric graph-based estimation. J Stat Softw 40:1-29

Edenbrow M, Croft DP (2012) Kin and familiarity influence association preferences and aggression in the mangrove killifish Kryptolebias marmoratus. J Fish Biol 80:503-518

Fiske P, Rintamäki PT, Karvonen E (1998) Mating success in lekking males: a metaanalysis. Behav Ecol 9:328-338

Gibson RM, Pirs D, Delaney KS, Wayne RK (2005) Microsatellite DNA analysis shows that greater sage grouse leks are not kin groups. Mol Ecol 14:4453-4459 
Gosling LM, Petrie M, Rainy ME (1987) Lekking in topi: a high cost, specialist strategy. Anim Behav 35:616-618

Grafen A (2006) Optimization of inclusive fitness. J Theor Biol 238:541-563

Griffin AS, West SA (2002) Kin selection: fact and fiction. Trends Ecol Evol 17:15-21

Hämäläinen A, Alatalo RV, Lebigre C, Siitari H, Soulsbury CD (2012) Fighting behaviour as a correlate of male mating success in black grouse Tetrao tetrix. Behav Ecol Sociobiol 66:1577-1586

Hamilton WD (1964) The genetical evolution of social behaviour I \& II. J Theor Biol 7:1-52

Hatchwell BJ (2010) Cryptic kin selection: kin structure in vertebrate populations and opportunities for kin-directed cooperation. Ethology 116:203-216

Höglund J (2003) Lek-kin in birds - provoking theory and surprising new results. Ann Zool Fenn 40:249-253

Höglund J, Alatalo RV (1995) Leks. Princeton University Press, Princeton

Höglund J, Alatalo RV, Lundberg A, Ratti O (1994) Context-dependent effects of tailornament damage on mating success in black grouse. Behav Ecol 5:182-187

Höglund J, Alatalo RV, Lundberg A, Rintamäki PT, Lindell J (1999) Microsatellite markers reveal the potential for kin selection on black grouse leks. Proc R Soc Lond B 266:813-816

Höglund J, Johansson T, Pelabon C (1997) Behaviourally mediated sexual selection: Characteristics of successful male black grouse. Anim Behav 54:255-264

Höglund J, Kålås JA, Fiske $P$ (1992) The costs of secondary sexual characters in the lekking great snipe (Gallinago media). Behav Ecol Sociobiol 30:309-315

Höner OP, Wachter B, East ML, Streich WJ, Wilhelm K, Burke T, Hofer H (2007) Female mate-choice drives the evolution of male-biased dispersal in a social mammal. Nature 448:798-801

Hood GM (2011) PopTools version 3.2.5, http://www.poptools.org

Hovi M, Alatalo RV, Höglund J, Lundberg A, Rintamäki PT (1994) Lek centre attracts black grouse females. Proc R Soc Lond B 258:303-305 
Hsu Y, Earley RL, Wolf LL (2006) Modulation of aggressive behaviour by fighting experience: Mechanisms and contest outcomes. Biol Rev 81:33-74

Isvaran K, Ponkshe A (2013) How general is a female mating preference for clustered males in lekking species? A meta-analysis. Anim Behav 86:417-425

Kervinen M, Alatalo RV, Lebigre C, Siitari H, Soulsbury CD (2012) Determinants of yearling male lekking effort and mating success in black grouse (Tetrao tetrix). Behav Ecol 23:1209-1217

Kervinen M, Lebigre C, Alatalo RV, Siitari H, Soulsbury CD (2015) Life history differences in age-dependent expressions of multiple ornaments and behaviors in a lekking bird. Am Nat $185: 13-27$

Kervinen M, Lebigre C, Soulsbury CD (2016) Simultaneous age-dependent and ageindependent sexual selection in the lekking black grouse (Lyrurus tetrix). J Anim Ecol $85: 715-725$

Kirkpatrick M, Ryan MJ (1991) The evolution of mating preferences and the paradox of the lek. Nature 350:33-38

Kokko H, Lindström J (1996) Kin selection and the evolution of leks: whose success do young males maximize? Proc R Soc Lond B 263:919-923

Kokko H, Lindström J, Alatalo RV, Rintamäki PT (1997) Queuing for territory positions in the lekking black grouse (Tetrao tetrix). Behav Ecol 9:376-383

Kokko H, Rintamäki PT, Alatalo RV, Höglund J, Karvonen E, Lundberg A (1999) Female choice selects for lifetime lekking performance in black grouse males. Proc R Soc Lond B 266:2109-2115

Komdeur J (1994) The effect of kinship on helping in the cooperative breeding Seychelles warbler (Acrocephalus sechellensis). Proc R Soc Lond B 256:47-52

Koprowski JL (1996) Natal philopatry, communal nesting, and kinship in fox squirrels and gray squirrels. J Mammal 77:1006-1016

Krakauer AH (2005) Kin selection and cooperative courtship in wild turkeys. Nature 434:6972 
Krützen M, Sherwin WB, Barre LM, Connor RC, Van de Casteele T, Mann J, Brooks R (2003) Contrasting relatedness patterns in bottlenose dolphins (Tursiops sp.) with different alliance strategies. Proc R Soc Lond B 270:497-502

Lebigre C, Alatalo RV, Forss HE, Siitari H (2008). Low levels of relatedness on black grouse leks despite male philopatry. Mol Ecol 17:4512-4521

Lebigre C, Alatalo RV, Kilpimaa J, Staszewski V, Siitari H (2012) Leucocyte counts variation and measures of male fitness in the lekking black grouse. J Ornithol 153:95-102

Lebigre C, Alatalo RV, Siitari H (2010) Female-biased dispersal alone can reduce the occurrence of inbreeding in black grouse. Mol Ecol 19:1929-1939

Lebigre C, Alatalo RV, Siitari H (2013) Physiological costs enforce the honesty of lek display in the black grouse (Tetrao tetrix). Oecologia 172:983-993

Lebigre C, Alatalo RV, Siitari H, Parri S (2007) Restrictive mating by females on black grouse leks. Mol Ecol 16:4380-4389

Lebigre C, Alatalo RV, Soulsbury CD, Höglund J, Siitari H (2014) Limited indirect fitness benefits of male group membership in a lekking species. Mol Ecol 23:5356-5365

Lion S, Gandon S (2009) Habitat saturation and the spatial evolutionary ecology of altruism. J Evol Biol 22:1487-1502

Loiselle BA, Ryder TB, Durães R, Tori W, Blake JG, Parker PG (2007) Kin selection does not explain male aggregation at leks of 4 manakin species. Behav Ecol 18:287-291

MacColl ADC, Piertney SB, Moss R, Lambin X (2000) Spatial arrangement of kin affects recruitment success in young male red grouse. Oikos 90:261-270

Magaña M, Alonso JC, Palacín C (2011) Age-related dominance helps reduce male aggressiveness in great bustard leks. Anim Behav 82:203-211

McElligott AG, Gammell MP, Harty HC, Paini DR, Murphy DT, Walsh JT, Hayden TJ (2001) Sexual size dimorphism in fallow deer (Dama dama): Do larger, heavier males gain greater mating success? Behav Ecol Sociobiol 49:266-272 
McElligott AG, Naulty F, Clarke WV, Hayden TJ (2003) The somatic cost of reproduction: What determines reproductive effort in prime-aged fallow bucks? Evol Ecol Res 5:1239_ 1250

Mitteldorf J, Wilson DS (2000) Population viscosity and the evolution of altruism. J Theor Biol $204: 481-496$

Parker GA, Sutherland WJ (1986) Ideal free distributions when individuals differ in competitive ability: phenotype-limited ideal free models. Anim Behav 34:1222-1242

Piertney SB, Lambin X, Maccoll ADC et al (2008) Temporal changes in kin structure through a population cycle in a territorial bird, the red grouse Lagopus lagopus scoticus. Mol Ecol 17:2544-2551

Piertney SB, MacColl ADC, Lambin X, Moss R, Dallas JF (1999) Spatial distribution of genetic relatedness in a moorland population of red grouse (Lagopus lagopus scoticus). Biol J Linn Soc 68:317-331

Pinheiro J, Bates D, DebRoy S, Sarkar D, R Core Team (2013) nlme: Linear and Nonlinear Mixed Effects Models. $\mathrm{R}$ package version 3.1-108, https://cran.rproject.org/web/packages/nlme/index.html

Pravosudova EV, Grubb TC, Parker PG (2001) The influence of kinship on nutritional condition and aggression levels in winter social groups of Tufted Titmice. Condor 103: $821-828$

Queller DC, Goodnight KF (1989) Estimating relatedness using genetic markers. Evolution $43: 258-275$

R Core Development Team (2012) R: A language and environment for statistical computing. R Foundation for Statistical Computing, Vienna, Austria, http://www.R-project.org/ Reeve HK, Westneat DF, Noon WA, Sherman PW, Aquadro CF (1990) DNA 'fingerprinting' reveals high levels of inbreeding in colonies of the eusocial naked mole-rat. P Natl Acad Sci USA 87:2496-2500 
Reynolds SM, Christman MC, Uy JAC, Patricelli GL, Braun MJ, Borgia G (2009) Lekking satin bowerbird males aggregate with relatives to mitigate aggression. Behav Ecol $20: 410-415$

Rintamäki PT, Höglund J, Alatalo RV, Lundberg A (2001) Correlates of male mating success on black grouse (Tetrao tetrix L.) leks. Ann Zool Fenn 38:99-109

Russell AF, Hatchwell BJ (2001) Experimental evidence for kin-biased helping in a cooperatively breeding vertebrate. Proc R Soc Lond B 268:2169-2174

Sæther SA (2002) Kin selection, female preference and the evolution of leks: direct benefits may explain kin structuring. Anim Behav 63:1017-1019

Segelbacher G, Wegge P, Sivkov AV, Höglund J (2007) Kin groups in closely spaced capercaillie leks. J Ornithol 148:79-84

Shorey L, Piertney SB, Stone J, Höglund J (2000) Fine-scale genetic structuring on Manacus manacus leks. Nature 408:352-353

Silk JB (2002) Kin selection in primate groups. Int J Primatol 23:849-875

Smith JE, Van Horn RC, Powning KS, Cole AR, Graham KE, Memenis SK, Holekamp KE (2010) Evolutionary forces favoring intragroup coalitions among spotted hyenas and other animals. Behav Ecol 21:284-303

Taylor P (1992) Altruism in viscous populations-an inclusive fitness model. Evol Ecol

$$
6: 352-356
$$

Templeton CN, Reed VA, Campbell SE, Beecher MD (2012) Spatial movements and social networks in juvenile male song sparrows. Behav Ecol 23:141-152

Van Dyken JD (2010) The components of kin competition. Evolution 64:2840-2854

Vehrencamp SL, Bradbury JW, Gibson RM (1989) The energetic cost of display in male sage grouse. Anim Behav 38:885-896

Wahaj SA, Van Horn RC, Van Horn TL, Dreyer R, Hilgris R, Schwarz J, Holekamp KE (2004) Kin discrimination in the spotted hyena (Crocuta crocuta): nepotism among siblings.

$$
\text { Behav Ecol Sociobiol 56:237-247 }
$$


547 Warren PK, Baines D (2002) Dispersal, survival and causes of mortality in black grouse

$548 \quad$ Tetrao tetrix in northern England. Wildlife Biol 8:91-97

549 West Eberhard MJ (1975) The evolution of social behavior by kin selection. Q Rev Biol 50:1$550 \quad 33$

551 Wilson DS, Pollock G, Dugatkin L (1992) Can altruism evolve in purely viscous populations? $552 \quad$ Evol Ecol 6:331-341

553 
$554 \quad$ Figure captions

555 Fig. 1 Distributions of the relatedness estimates for all neighbouring males (panel A) and

556 number of close relatives to newly territorial males (panel B). Close relatives were

557 conservatively defined as having a pairwise relatedness coefficient superior to 0.2

558 Fig. 2 Effect of the territory centrality difference on the fight frequency of neighbouring males

559 Fig. 3 Mean relatedness of newly territorial males to their neighbours and to the other lekking 560 males 